\title{
Mastitis associated with Prototheca zopfii - an emerging health and economic problem on dairy farms
}

\author{
Dubravka Milanov $^{1}$, Tamaš Petrović ${ }^{1}$, Vladimir Polaček ${ }^{1}$, \\ Ljiljana Suvajdžić ${ }^{2}$, Jovan Bojkovski ${ }^{3}$ \\ ${ }^{1}$ Scientific Veterinary Institute "Novi Sad", 21000 Novi Sad, Republic of Serbia \\ ${ }^{2}$ Department of Pharmacy, Faculty of Medicine, \\ University of Novi Sad, 21000 Novi Sad, Republic of Serbia \\ ${ }^{3}$ Faculty of Veterinary Medicine, University of Belgrade, 11000 Belgrade, Republic of Serbia \\ dubravka@niv.ns.ac.rs
}

Received: May 19, $2016 \quad$ Accepted: November 16, 2016

\begin{abstract}
Increased incidence of protothecal mastitis has been recorded in several countries in the past ten years. The main goal of this article is to draw the attention of scientific and professional community to the emerging issue of mammary protothecosis. The article collates currently known facts about infection reservoirs, predisposing factors for the development of mastitis, clinical manifestations of the disease, and potential transmission routes within the herd as well as the measures for control and eradication. We would like to point out that identification of protothecal mastitis on a dairy farm is associated with a range of problems. Early detection of infected animals can be difficult because of predominantly subclinical course of early-stage infection, which easily spreads between cows via the milking system. Spontaneous recovery has not been recorded and infected cows typically develop chronic mastitis with granulomatous infiltration and progressive loss of functional parenchyma of the mammary gland. Substantial economic losses and health damages associated with mammary protothecosis strongly emphasise the need for developing effective prevention strategies aimed at control of the infection.
\end{abstract}

Keywords: cows, Prototheca zopfii, mastitis.

\section{Introduction}

The algae from the genus Prototheca are closely related to the Chlorella sp., green algae from the same family of Chlorellaceae; however, Prototheca has lost its ability to synthesise chlorophyll and adopted a heterotrophic mode of nutrition $(12,16,26)$. The genus Prototheca encompasses six generally accepted species: $P$. stagnora, $P$. ulmea, $P$. blaschkeae, $P$. wickerhamii, $P$. zopfii, and $P$. cutis sp. nov. $(8,12$, 30). The species $P$. blaschkeae, $P$. wickerhamii, $P$. zopfii, and $P$. cutis are considered causal agents of local human and/or animal infections $(18,21,28,30$, 31). Systemic infections associated with these organisms are relatively rare.

According to its assimilation patterns $P$. zopfii has been subdivided into three biotypes or 'variants': 1, 2 , and 3. Biotype 2 is characterised by delayed assimilation of galactose and increased assimilation of amino acids in comparison to biotype 1 , whilst the strains of biotype 3 are incapable of glycerol assimilation (27). P. zopfii biotype 2 has been established to be the major causative agent of mastitis in dairy cows $(3,9,14,22,23,29)$. Genotype 1 is considered to be non-pathogenic (12). Roesler et al. (28) showed that the three biotypes clearly differ in both the small-subunit (18S) rRNA gene and cellular fatty acid composition. It has been proven that partial sequences (1442 bp) of the 18S rRNA genes differ between the strains of the three biotypes, and all the strains of a certain biotype belong to the same species. The sequences of biotype 3 differed from the others and had a sequence similarity of $98.1 \%$. Also, there was a difference between the sequences of biotype 2 and biotype 1 , with a similarity of $99.4 \%$. Thus, the phylogenetic distance is the largest between strains of 
$P$. zopfii biotype 3 and the biotypes 1 and 2 (28). Later on, two novel genotypes 1 and 2 were proposed, whereas biotype 3 was categorised as the new species Prototheca blaschkeae sp. nov. (22, 28).

$P$. zopfii was first identified as a causative agent of bovine mastitis in Germany in 1952 (17). So far, $P$. zopfii has been isolated worldwide from milk of cows with clinical and subclinical mastitis $(1,2,6,17)$. In Serbia, protothecal mastitis has been first diagnosed in 2006 on a Holstein-Friesian dairy farm in Vojvodina (20). Isolation of $P$. blaschkeae and $P$. wickerhamii has also been reported; however, the organisms are considered rare causal agents of bovine mastitis (7, 14, 29).

Throughout the past few years, substantial increase in incidence of $P$. zopfii mastitis has been reported all over the world (3, 9, 10, 16, 26, 29, 31). Since 2006, P. zopfii has been isolated from milk on four dairy farms in the regions of Vojvodina and Central Serbia; however, its much wider prevalence is suspected in some regions of Serbia even though it has not yet been isolated. The objective of this article is to draw the attention of scientific and professional community to the growing problem of $P$. zopfii mastitis and its clinical, epizootiological, and diagnostic aspects as well as potential human health implications.

\section{Common habitats of $P$. zopfii and the potential source of infection for cows}

$P$. zopfii is widely distributed in the nature. Humid areas, particularly these containing manure and decomposing plant material, are its natural habitat (26). As the diet of dairy cattle is based on feed of plant origin, the algae may be a transient flora in the gastrointestinal tract of cows and as such are excreted intact (17). As a consequence of recent global climate changes, high temperatures, often combined with high humidity, enhance the multiplication of the algae in the environment $(4,7,13,31)$. $P$. zopfii is a unicellular alga that reproduces asexually by formation of 2-16 daughter cells (endospores) inside the mother cell (sporangia) (25). The rupture of the sporangia releases the endospores that start the new cycle of their development into mature sporangia (Fig. 1). The reproduction cycle takes place at higher temperatures, with the lowest temperature limit estimated at about $25^{\circ} \mathrm{C}(10,26)$.

Along with the majority of other mastitic agents, $P$. zopfii is assigned to a group of environment-borne pathogens. Identification of potential infection sources on the farm is useful in view of implementation of relevant preventive measures; however, the real source of infection often remains unknown (13). At a dairy farm with protothecal mastitis, Osumi et al. (23) isolated $P$. zopfii from drinking water, bovine faeces, sewage water, and the milk of infected cows. The analysis using genotype-specific PCR assays and restriction fragment length polymorphism (RFLP) test revealed $P$. zopfii genotype 2 in all isolates from cow milk $(n=67)$. Still, out of 32 isolates collected from the environment as many as 29 were identified as genotype 1, whereas only three isolates belonged to genotype 2 (23). Specific reservoir of $P$. zopfii in the environment has not been identified.

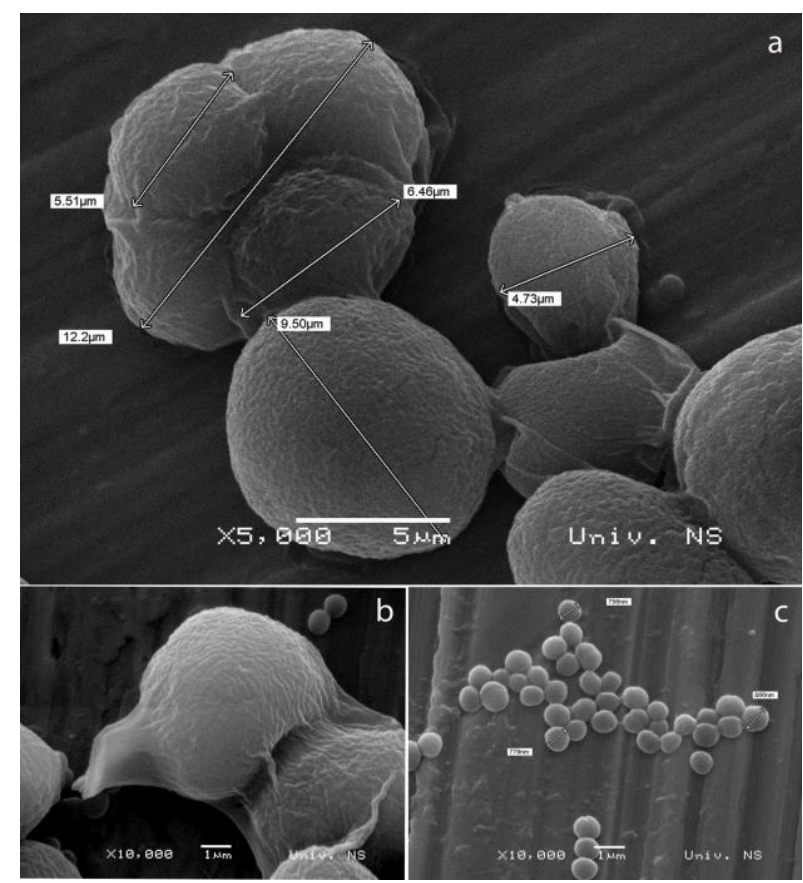

Fig. 1. Scanning electron microscopy of $P$. zopfii isolates from cow's milk: a - sporangia of Prototheca zopfii (7-16 $\mu \mathrm{m}) ; \mathrm{b}$ and c - comparison of the size of P.zopfii spores with the cells of Staphylococcus sp. (up to $1 \mu \mathrm{m}$ ) (Milanov D., Scientific Veterinary Institute „Novi Sad“; scanning electron microscopy: Prof Dr Bokorov M., Faculty of Sciences, University of Novi Sad)

Infected cows cannot be excluded as the possible sources of infection for other animals. P. zopfii was isolated from milking machine liners and milking cups (16). The organism can be transferred from infected cows to other animals in the same way as any other agent of contagious mastitis (2).

\section{Predisposing factors for the development of $P$. zopfii mastitis}

P. zopfii is commonly detected in cow faeces and in a range of environmental sources even on farms with no history of protothecal mastitis $(2,17)$. Ubiquitous distribution of the organism in the nature and relatively low pathogenic potential of $P$. zopfii underline the role of predisposing factors in the development of mastitis. Identification of relevant risk factors is of major importance in the control of the disease; however, causal relationships between various factors in each individual case are still difficult to establish. Poor hygiene practices, excessive antibiotic use, and inadequate milking hygiene are most commonly considered the major risk factors for mastitis development $(10,13,17,24,26,31)$. 
Pieper et al. (24) have conducted extensive research aimed at identifying cow- and herd-level risk factors for the occurrence of Prototheca mastitis. The research encompassed 23 Holstein-Friesian farms in Ontario (Canada). Prototheca spp. was isolated from 64 milk samples from cows originating from 18 farms with an average prevalence of $5.1 \%$ (range $0.0 \%-$ $12.5 \%$ ). The authors believe that the actual prevalence of Prototheca spp. in positive herds and animals was even higher than the one determined in this study, because cows that were dry at the time of the investigation were not tested. Moreover, intermittent shedding of the algae in milk may prevent detection of the agent during one single testing or examination of lactating cows. Pieper et al. (24) established that unsanitary or repeated intramammary infusions and antibiotic treatment can contribute to the development of Prototheca infection of the mammary gland. Similarly to other pathogens manifesting natural resistance to antibiotics, inadequate intramammary administration of antibiotics (contaminated applicator or inadequate teat disinfection before administration) is an important risk factor for direct introduction of the organism into the mammary gland. Excessive usage of antibiotics and consequent elimination of the competitive natural udder flora can give rise to the development of algal infections. Interestingly, specific farm management practices or milking hygiene deficiencies were not identified as risk factors for occurrence of Prototheca mastitis in the aforementioned study (24).

\section{Development of mammary infection with $P$. zopfii}

Intramammary protothecosis always develops as an ascending infection (15). The cows are susceptible to infection disregarding the age and lactation stage. Protothecal mastitis frequently affects primiparous cows, but can also develop during the dry periods. Cows in early lactation are particularly susceptible, which is due to energy imbalance and physiologically reduced immunity $(13,20,31)$. An open teat sphincter as well as some small teat lesions caused by milking equipment constitute a potential portal of entry of the infection (11).

In the dry period, especially immediately before and after parturition, the mammary gland is highly susceptible to infections caused by environmental microorganisms (5). Thus, mastitis control programmes usually include intramammary application of antibiotics which are to eliminate the existing infections and to prevent the potential new infections. However, not only may the expected effects be absent (5), but such intrammamary administration may involve the risk of iatrogenic contamination and direct entrance of Prototheca or other pathogens into the mammary gland.

\section{Clinical manifestations of $P$. zopfii mastitis}

P. zopfii mastitis can occur as sporadic infection, but endemic forms have also been reported (13, 15, 20). The inflammatory process can affect individual quarters as well as the entire udder. At the outset, the infection is commonly asymptomatic. Contrary to infections caused by other mastitic pathogens, the initial mammary immune response to protothecal infection is very mild, and the somatic cell count (SCC) usually remains unchanged during the initial stages of the disease (31). In cows infected with $P$. zopfii, the SCC in quarter and composite milk samples can be lower than 100,000 cells/mL (24). The infection is commonly subclinical and is discovered only after significant increase of the SCC (8). If the composite milk sample is tested for the SCC, the infection of individual quarters can remain unnoticed. Even significant increase in the SCC can be missed in composite milk sample because of mixing (i.e. diluting) with milk from healthy quarters. Throughout the further course of the disease, the number of somatic cells increases from $500,000 / \mathrm{mL}$ (11) to even several millions $(13,31)$. The SCC value in milk originating from a cow with clinical protothecal mastitis can exceed 20 million/mL (17).

Clinically manifested infections are associated with changes in the appearance and consistency of milk. It becomes watery, grayish in colour, with or without small flakes (Fig. 2). Udder pain and oedema can occur in some animals $(17,31)$. Absence of systemic infection manifestations has been commonly observed (14), whereas increased body temperature and appetite loss were rarely reported (31).

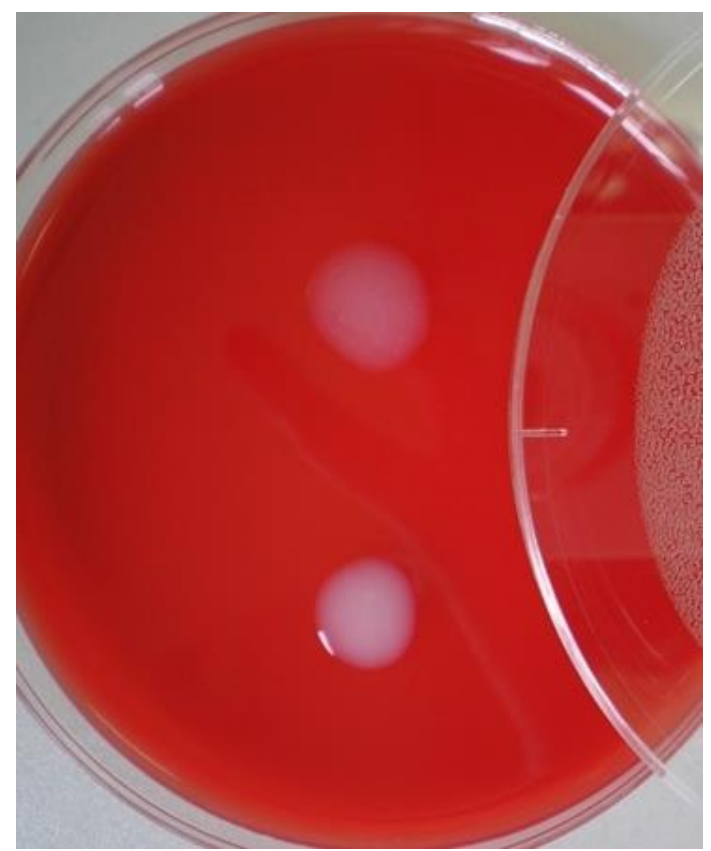

Fig. 2. Appearance of a milk drop originating from cow with protothecal mastitis (upper) compared with milk of healthy cow (lower) on blood agar (Milanov D., Scientific Veterinary Institute ,Novi Sad“) 
Protothecal mastitis is not a self-limiting infection and does not cure spontaneously. The infection becomes chronic and $P$. zopfii persists inside the macrophages in the udder through several lactating and dry periods (13). In turn, milk production decreases while the SCC increases $(4,31,32)$. Local immune response results in granulomatous proliferation and irreversible atrophy of functional parenchyma $(13,20$, 31). Histopathological examination of the mammary gland reveals the presence of $P$. zopfii organisms in the alveolar lumen, interstitium, neutrophils, and macrophages $(17,31)$. Our research conducted on the dairy farm in Serbia, where we first diagnosed $P$. zopfii mastitis in 2006, revealed pronounced enlargement and hard consistency (stiffness) of infected udders of diseased animals. In individual animals, chronic infection resulted in highly severe granulomatous proliferations which even caused rupture of udder skin due to over-stretching. Such situation on the farm is likely due to a range of previous misdiagnoses and consequent prolonged inadequate antibiotic treatments.

So far, virulence factors of $P$. zopfii as well as the differences in pathogenic potential of various Prototheca species and genotypes have not been fully elucidated (12). Thus, the course and pathogenesis of protothecal diseases still remain unclear.

\section{Diagnosis of $P$. zopfii mastitis}

An accurate diagnosis of protothecal mastitis relies on isolation and identification of the agent (26, 31). If protothecal mastitis has been established in the herd, immediate examination of milk from all cows is of crucial importance for detecting early and subclinical infections. Milk samples from individual quarters should be examined to avoid diagnostic failure. Namely, if only one quarter is infected, dilution with milk from healthy, uninfected quarters will decrease the pathogen count in composite milk sample below the limit of detection (24). Intermittent shedding the organism by infected cows calls for regular repeated testing $(10,24)$. Advanced stages of the disease are characterised by highly elevated numbers of algae in milk of infected cows, and laboratory diagnosis is quite simple to establish.

$P$. zopfii grows readily on conventional laboratory media such as 5\% sheep blood agar, and MacConkey and Sabouraud dextrose agars. Prototheca is a slowgrowing alga, thus requiring a two- to three-day incubation period on 5\% sheep blood agar. After 48$72 \mathrm{~h}$ incubation on blood agar, Prototheca zopfii forms distinct small gray colonies of around $1 \mathrm{~mm}$ in diameter, without haemolysis. Colonies grown on MacConkey agar are extremely small. The growth of $P$. zopfii on Sabouraud dextrose agar can be observed after $24 \mathrm{~h}$ of incubation at $37^{\circ} \mathrm{C}$ (Fig. 3a, left). The colonies are usually smaller as compared with those of Candida species, but after $48 \mathrm{~h}$ they are clearly visible, white to cream-coloured, 3-4 $\mathrm{mm}$ in diameter (Fig. 3a, right). $P$. zopfii grows at incubation temperatures between 25 and $37^{\circ} \mathrm{C}$.

Isolation and identification of $P$. zopfii from cow milk has not yet been introduced into routine practice of many microbiology laboratories in Serbia. Moreover, the herd veterinarians are not wellacquainted with the clinical and epizootiologic characteristics of protothecal mastitis. Dairy farms can suffer substantial economic losses due to ill-timed suspicion of Prototheca infection, and hence the lack of early and accurate aetiological diagnosis. Protothecal mastitis can result in culling of the entire herd as it was the case in 2006, at the aforementioned HolsteinFriesian dairy farm in Serbia. During 2015, we found an endemic form of protothecal mastitis on a dairy farm in Serbia. More than 30 high-yielding dairy cows were culled from the herd, while microbiological examination revealed subclinical $P$. zopfii infection in 12 more cows. Regrettably, throughout the preceding period of more than one year, milk samples from this farm had been regularly submitted for testing to the regional veterinary microbiology laboratory (because of history of positive California mastitis test) where $P$. zopfii had been misidentified as Candida spp.

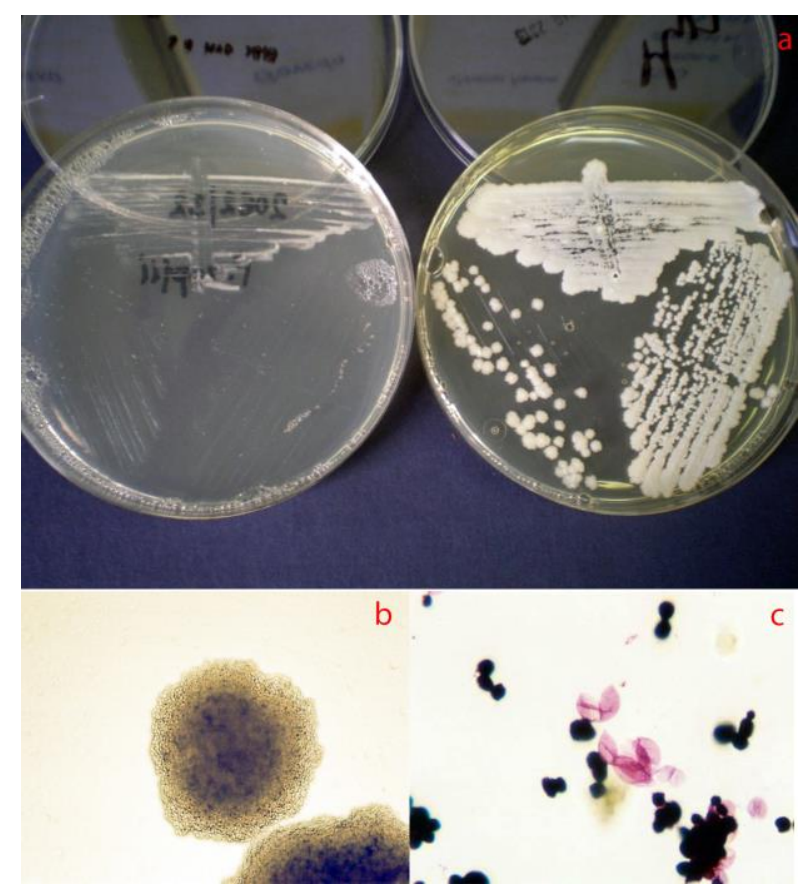

Fig. 3. Cultural and microscopic characteristics of $P$. zopfii isolates from cow's milk. a - Prototheca zopfii colonies on Sabouraud dextrose agar after $24 \mathrm{~h}$ (left) and $48 \mathrm{~h}$ (right) of incubation at $37^{\circ} \mathrm{C}$; b - colonial appearance of Prototheca zopfii (light microscopy, $200 \mathrm{x}$ ); c - Gram-negative empty sporangia and Gram-positive spores of Prototheca zopfii (light microscopy, 1000 x). Photo: Milanov D., Scientific Veterinary Institute „Novi Sad““

P. zopfii differs from Candida species by its colonial appearance, though comparison of these two microbial species commonly relies on their growth characteristics. We are of the opinion that the similarity applies only to more intensive growth on Sabouraud dextrose agar as compared with some other culture media for isolation of mastitic agents (5\% sheep blood 
agar or MacConkey agar). Low magnification of light microscopy revealed characteristic granulated appearance of $P$. zopfii colonies on Sabouraud dextrose agar (Fig. 3b), which has not been observed in yeast cultures in our practice so far. Gram-staining of the microscopic preparation clearly distinguished the algae from yeasts. The preparations revealed Gram-positive spores and Gram-negative sporangia (Fig. 3c). Morphological features, i.e. the structure of sporangia at different stages of sporulation process, are easily perceptible by examining a fresh drop of colonial suspension in one drop of Lugol's solution, lactophenol, toluidine blue, or in preparations stained with methylene blue, malachite green, Congo red, or fuchsine (26). Once transferred into the liquid, P. zopfii disperses easily and uniformly, which is not typical for yeasts. A range of commercial biochemical tests such as API $20 \mathrm{C}$ and ID32 systems are available for rapid differentiation of algae from yeasts (26). The main drawback of the standard method of isolation and identification of $P$. zopfii is its duration - it usually takes more than three days. Thus, molecular techniques (PCR) for prompt identification of Prototheca zopfii genotypes 2 directly from milk samples are available today $(8,9)$. It has been proven that PCR- and RFLPassays may distinguish between pathogenic and apathogenic strains of $P$. zopfii and between these and $P$. blaschkeae, a putative milk contaminant (22).

\section{Treatment of $P$. zopfii mastitis}

Prototheca mastitis is an incurable infection. In vitro, $P$. zopfii isolates from cow milk manifest susceptibility towards antifungal agents such as amphotericin B and nystatin, and even towards some antibiotics such as gentamicin, neomycin, and streptomycin. Wawron et al. (32) established resistance to clotrimazole, fluconazole, econazole, and flucytosine in all strains of $P$. zopfii $(\mathrm{n}=27)$ isolated from the milk of cows from one herd in the north-eastern part of Poland. We found nystatin, ketoconazole and amphotericin B $(88.9 \%$ and $0.0 \%, 51.9 \%$ and $22.2 \%$, $0.0 \%$ and $48.1 \%$ of susceptible and intermediate susceptible isolates respectively) as well as gentamicin (96.3\%), kanamycin (92.6\%), and polymyxin B $(59.3 \%)$ to be effective against $P$. zopfii. However, in vitro susceptibility of Prototheca isolates does not correlate with the effectiveness of antifungal agents in vivo. Mastitis is commonly left untreated and infected animals should be removed into a separate group and milked as last until culled from the herd.

Current research is aimed at the identification of immunogenic proteins of $P$. zopfii and the development of immunoprophylaxis. Due to the absence of effective treatment of prototheca infections, immunisation and immune therapy are of utmost importance. Protein extracts may be used to induce the host's immune response. For example, Hsp70 protein is considered the major antigen of $P$. zopfii genotype 2, responsible for a strong pro-inflammatory response (12). Knowledge on the sequence of the Prototheca genome will enable the understanding of the pathways of infection and a specific immunisation based on recombinant proteins.

\section{Consumption of milk and dairy products from infected cows may pose a risk for human health}

Human health concerns exist to some degree with respect to consumption of pasteurised milk or dairy products. $P$. zopfii is resistant to pasteurisation, and its potential to cause human enteritis has been reported (3, 12, 19)

\section{Concluding remarks}

Mastitis associated with $P$. zopfii cause substantial health and economic damage on dairy farms. Thus, efforts should be made to implement effective prevention strategies which would enable putting the disease under control. Good farm management practices, well-balanced nutrition of dairy cows, as well as adequate disinfection of milking equipment are the prerequisites for the accomplishment of this goal. Regular microbiological examination of milk samples from all cows is crucial for timely detection of the infection. The method of isolation and identification of $P$. zopfii has to be implemented into routine practice of laboratories involved in identification of mastitic agents. Furthermore, awareness of field veterinarians and their knowledge on clinical and epizootiologic features of protothecal mastitis are of the highest importance. In subclinical mastitis associated with increased SCC or mastitis that is irresponsive to antimicrobial therapy, timely suspicion of an algal infection is of crucial importance. Excessive empirical use of antibiotics should be avoided whenever possible, only in incident cases when apparent clinical manifestations require prompt intervention. Ideally, the therapy should be based on the results of laboratory identification of the causal agent of mastitis and the susceptibility of isolated bacterial species to antibiotics. In the case of protothecal or Candida mastitis the administration of antibiotics is useless, even counterproductive, and can be considered a misuse.

Diagnosis of protothecal mastitis in a herd is considered a serious problem, especially in cases of late diagnosis, i.e. disseminated infection within the herd. Reduced milk production, increased SCC, lack of effective treatment, and unavoidable culling of infected cows from the herd can result in enormous health and economic losses. Control of the infection is a highly demanding procedure because of the high prevalence of the agent in the environment and spreading of the infection within the herd via milking equipment. So far, elimination of infected animals is necessary and is known to be the most effective method for disease control. Some recent attempts aiming at prevention and 
control of protothecal mastitis are focused on immunodominant proteins of $P$. zopfii and potential immunisation based on recombinant proteins (12).

Conflict of Interests Statement: The authors declare that there is no conflict of interests regarding the publication of this article.

Financial Disclosure Statement: This paper is supported by a grant from the Ministry of Education, Science and Technological Development, Republic of Serbia, Project number TR 31071.

\section{References}

1. Aalbaek B., Jensen H.E., Huda A.: Identification of Prototheca from bovine mastitis in Denmark. APMIS 1998, 106, 483-486.

2. Anderson K.L., Walker R.L.: Sources of Prototheca spp. in a dairy herd environment. J Am Vet Med Assoc 1988, 193, 553-556.

3. Bozzo G., Bonerba E., Di Pinto A., Bolzoni G., Ceci E., Mottola A., Tantillo G., Terio V.: Occurrence of Prototheca spp. in cow milk samples. New Microbiol 2014, 37, 459-464.

4. Bueno V.F., de Mesquita A.J., Neves R.B., de Souza M.A., Ribiero A.R., Nicolau E.S., de Oliveira A.N.: Epidemiological and clinical aspects of the first outbreak of bovine mastitis caused by Prototheca zopfii in Goias State, Brazil. Mycopathologia 2006, 161, 141-145.

5. Cengiz M., Bastan A.: Effectiveness of dry cow therapy comprising antibiotic treatment, internal teat sealant, and $\alpha$-tocopherol against new intramammary infections in cows. Bull Vet Inst Pulawy 2015, 59, 71-78.

6. Costa E.O., Ribeiro A.R., Watanabe E.T., Pardo R.B., Silva J.A.B., Sanches R.B.: An increase incidence of mastitis caused by Prototheca species and Nocardia species on a farm in Sao Paulo, Brazil. Vet Res Commun 1996, 20, 237-241.

7. Costa E.O., Ribeiro A.R., Watanabe E.T., Melville P.A.: Infectious bovine mastitis caused by environmental organisms. Zentralbl Veterinarmed (B) 1998, 45, 67-71.

8. Cremonesi P., Pozzi F., Ricchi M., Castiglioni B., Luini B., Chessa S.: Identification of Prototheca species from bovine milk samples by PCR-single strand conformation polymorphism. J. Dairy Sci 2012, 95, 6963-6968.

9. Gao, J., Hou, R.G., Zhang, H.Q., He, J.Z., Li, S.M., Su, J.L., Han, B.: A novel DNA extraction and duplex polymerase chain reaction assay for the rapid detection of Prototheca zopfii genotype 2 in milk. Lett Appl Microbiol, 2011, 53, 278-282.

10. Gonçalves J.L., Lee S.H.I., de Paula Arruda E., Galles D.P, Caetano V.C., de Oliveira C.A.F, Fernandes A.M., Santos M.V.: Biofilm-producing ability and efficiency of sanitizing agents against Prototheca zopfii isolates from bovine subclinical mastitis. J Dairy Sci 2015, 98, 1-9.

11. Hodges R.T., Holland J.T.S., Neilsont F.J.A., Wallace N.M.: Prototheca zopfii mastitis in a herd of dairy cows. New Zeland Vet J 1985, 33, 108-111.

12. Irrgang A., Murugaiyan J., Weise C., Azab W., Roesler U.: Well-known surface and extracellular antigens of pathogenic microorganisms among the immunodominant proteins of the infectious microalgae Prototheca zopfii. Front Cell Infect Microbiol 2015, 5, 67, doi: 10.3389/fcimb.2015.00067.

13. Jánosi S., Szigeti G., Rátz F., Laukó T., Kerényi J., Tenk M., Katona F., Huszenicza A., Kulcsár M., Huszenicza G.: Prototheca zopfii mastitis in dairy herds under continental climatic conditions. Vet Q 2001, 23, 80-83.

14. Jánosi S., Rátz F., Szigeti G., Kulcsar M., Kerenyi J., Laukó T., Katona F., Huszenicza G.: Review of the microbiological, pathological and clinical aspects of bovine mastitis caused by the alga Prototheca zopfii. Vet Q 2001, 23, 58-61.

15. Jensen H.E., Aalbaek B., Bloch B., Huda A.: Bovine mammary protothecosis due to Prototheca zopfii. Med Mycol 1998, 36, 89-95.

16. Krukowski H., Lisowski A., Nowakowicz-Debek B., Wlazlo Ł.: Susceptibility of Prototheca zopfii strains isolated from cows with mastitis to chlorhexidine and iodine. Turk J Vet Anim Sci 2013, 37, 1110-1143.

17. Malinowski E., Lassa H., Klossowska A.: Isolation of Prototheca zopfii from inflamed secretion of udders, Bull Vet Inst Pulawy 2002, 46, 295-299.

18. Marques S., Silva E., Kraft C., Carvalheira J., Videira A., Huss V.A.R., Thompson G.: Bovine mastitis associated with Prototheca blaschkeae. J Clin Microbiol 2008, 46, 1941-1945.

19. Melville P.A., Watanabe E.T., Benites N.R., Ribeiro A.R., Silva J.A., Garino Junior F., Costa E.O.: Evaluation of the susceptibility of Prototheca zopfii to milk pasteurization. Mycopathologia 1999, 146, 79-82.

20. Milanov D., Suvajdžić L., Pušić I., Vidić B., Đorđević-Milić V.: Outbreak of endemic form of protothecal mastitis on a dairy farm. Acta Vet Beograd 2006, 56, 259-265.

21. Milanov D., Suvajdžić Lj.: Characteristics and importance of the genus Prototheca in human and veterinary medicine. Proc Natural Sci Matica Srpska Novi Sad 2006, 110, 15-27.

22. Möller A., Truyen U., Roesler U.: Prototheca zopfii genotype 2 the causative agent of bovine protothecal mastitis? Vet Microbiol 2007, 120, 370-374.

23. Osumi T., Kishimoto Y., Kano R., Maruyama H., Onozaki M., Makimura K., Ito T., Matsubara K., Hasegawa A.: Prototheca zopfii genotypes isolated from cow barns and bovine mastitis in Japan. Vet Microbiol 2008, 131, 419-423.

24. Pieper L., Godkin A., Roesler U., Polleichtner A., Slavic D., Leslie K.E, Kelton D.F.: Herd characteristics and cow-level factors associated with Prototheca mastitis on dairy farms in Ontario, Canada. J Dairy Sci 2012, 95, 5635-5644.

25. Pore R.S., Barnet E.A., Barnes W.C. Jr, Walker J.D.: Prototheca ecology. Mycopathologia 1983, 81, 49-62.

26. Rapuntean S., Rapuntean G., Fit N., Cuc C., Nadas G.: Morphological and cultural characterization of some strains of unicellular algae of the genus Prototheca sampled from mastitic cow milk. Notulae Botanicae Horti Agrobotanici Cluj-Napoca 2009, 37, 31-40.

27. Roesler, U., Scholz, H., Hensel, A. Emended phenotypic characterization of Prototheca zopfii: a proposal for three biotypes and standards for their identification. Int $\mathbf{J}$ Syst Evol Microbiol 2003, 53, 1195-1199.

28. Roesler U., Möller A., Hensel A., Baumann D., Truyen U.: Diversity within the current algal species Prototheca zopfii: a proposal for two Prototheca zopfii genotypes and description of a novel species, Prototheca blaschkeae sp. nov. Int J System Evol Microbiol 2006, 56, 1419-1425.

29. Salerno T., Ribeiro M.G., Langoni H., Siqueira A.K., da Costa E.O., Melville P.A., Bueno V.F.F., Yamamura A.A.M, Roesler U., da Silva A.V.: In vitro algaecide effect of sodium hypochlorite and iodine based antiseptics on Prototheca zopfii strains isolated from bovine milk, Res Vet Sci, 2010, 88, 211213.

30. Satoh K., Ooe K., Nagayama H., Makimura K.: Prototheca cutis sp. nov., a newly discovered pathogen of protothecosis isolated from inflamed human skin. Int J Syst Evol Microbiol 2010, 60, 1236-1240.

31. Wawron W., Bochniarz M., Piech T., Łopuszyński W., Wysocki J.: Outbreak of protothecal mastitis in a herd of dairy cows in Poland. Bull Vet Inst Pulawy 2013, 57, 335-339.

32. Wawron W., Bochniarz M., Piech T., Wysocki J., Kocik M.: Antimicrobial susceptibility of Prototheca zopfii isolated from bovine mastitis. Bull Vet Inst Pulawy 2013, 57, 485-488. 